\title{
Aproximación al manejo de la enfermedad de Freiberg
}

\author{
Approach to managing the Freiberg's disease
}

\author{
Daniel López López", Patricia Palomo López ${ }^{* *}$, Sergio Pérez García ${ }^{* * * *}$, \\ Rubén SÁNChez Gómez ${ }^{* * * *}$
}

* PhD. Doctor por la Universidade da Coruña. Profesor Asociado. Departamento de Ciencias da Saúde. Facultad de Enfermería y Podología. Universidade da Coruña.

daniellopez@udc.es

** PhD. Doctor por la Universidad Rey Juan Carlos. Profesora Colaboradora. Departamento de Enfermería. Centro Universitario de Plasencia. Universidad de Extremadura. patibiom@unex.es

*** Diplomado Universitario en Podología por la Universidade da Coruña. Doctorando del Programa de Ciencias Sociosanitarias. Universidade da Coruña. wilmoth_182@hotmail.com

**** PhD. Doctor por la Universidad Complutense de Madrid. Profesor Universidad Europea de Madrid. ruben.sanchez@reebokclub.com

Correspondencia

Prof. Dr. Daniel López López

Universidade da Coruña

Departamento de Ciencias da Saúde

Facultad de Enfermaría e Podoloxía

Campus Universitario de Esteiro s/n

15403 Ferrol (España)

E-mail: daniellopez@udc.es

Fecha de recepción: 10 octubre de 2011

Fecha de aceptación: 22 de febrero de 2012

Los autores declaran no tener ningún tipo de interés económico o comercial.

\section{RESUMEN}

La enfermedad de Freiberg, descrita por Albert H. Freiberg en 1914, es una osteocondrosis que se presenta en los metatarsianos y con mayor frecuencia en la segunda cabeza metatarsal.

La carencia de consenso en relación a la etiología multifactorial que presenta esta afección y la prevalencia de esta enfermedad a nivel del pie, sobre todo en la adolescencia y en deportistas, nos lleva a mejorar los conocimientos existentes en lo que se refiere a su clasificación, diagnóstico y tratamiento en la búsqueda de actualizar los conceptos relacionados con esta patología del pie.

Palabras clave: necrosis avascular; metatarsalgia; infracción de freiberg; osteocondritis.

\section{ABSTRACT}

Freiberg's disease, described by Albert H. Freiberg in 1914, is presented in osteochondrosis metatarsals and more frequently in the second metatarsal head.

The lack of consensus regarding the multifactorial etiology has this condition and the prevalence of this disease at the foot, especially in adolescence and athletes, leads us to improve existing knowledge in regard to their classification, diagnosis and treatment in the quest to update the concepts related to this foot disorders.

Key words: avascular necrosis; metatarsalgia; freiberg infraction; osteochondritis.

Sumario: 1. Introducción, 2. Etiología, 3. Clínica, 4. Diagnóstico, 5. Diagnóstico diferencial, 6. Tratamiento, 7. Conclusiones, Bibliografía.

Referencia normalizada: López López, D., Palomo López, P., Pérez García, S., Sánchez Gómez, R. Aproximación al manejo de la enfermedad de Freiberg. Rev. Int. Cienc. Podol. 2013; 7(1): 33-40. 


\section{INTRODUCCIÓN}

La enfermedad de Freiberg es una patología de antepie de consulta habitual por parte de las personas que acuden al podólogo. El lugar usual de aparición de la afección se relaciona con la cabeza de los metatarsianos y de forma especial a la cabeza del segundo y / o tercero, que se caracteriza por la pérdida temporal o permanente del flujo sanguíneo, conocida como osteonecrosis o necrosis avascular y que en las pruebas anatomopatológicas se observa un colapso en el hueso subcondral, en el tejido cartilaginoso y se aprecian zonas de fisuras óseas ${ }^{1,2}$.

Por este motivo y debido a la alteración en la condrogénesis y en la osteogénesis en la cabeza metatarsal del hueso, que previamente es normal se conoce a esta enfermedad también como Infracción de Freiberg (fractura incompleta), que está relacionada con la osteonecrosis que se produce en la zona afectada de lesión ${ }^{3,4}$.

La descripción de esta enfermedad le pertenece a Freiberg, que en el año 1914 informa del hallazgo patológico, relacionado con un doloroso hundimiento de la superficie articular de la cabeza del segundo metatarsiano y desde esa época se publican diferentes artículos que relatan las causas probables de su etiología, sin que a la fecha se llegara a un consenso en relación a la etiología real de este trastorno en el pie $^{5,6}$.

El primer artículo publicado por Freiberg, describe seis casos de mujeres jóvenes que presentaban cojera dolorosa y malestar aislado en la cabeza del segundo metatarsiano. En el examen radiológico, se observaba colapso de la superficie articular de la cabeza del segundo metatarsiano, con cuerpos sueltos intraarticulares visto en tres de los seis pacientes, relacionándolo con un primer radio insuficiente que provocaba un aumento de presión en la segunda articulación metatarsofalángica en carga $^{7}$.

El interés de esta patología se centra, en que es una de las afecciones que originan metatarsalgia siendo una de los principales motivos de consulta podológica y establecer el diagnóstico en las etapas iniciales puede ser difícil, debido a la etiología multifactorial asociada a traumas, déficit vascular y alteraciones sistémicas ${ }^{8,9}$.
Además genera pérdida de autonomía y deterioro en la calidad de vida debido a la limitación funcional que provoca en el pie a nivel especial y la repercusión en la salud a nivel general.

\section{ETIOLOGÍA}

La causa de inicio de la enfermedad es controvertida y en la actualidad se relaciona con un origen multifactorial, donde existen posibles factores etiológicos como ${ }^{10}$ :

- Alteración de la vascularización: las arterias radiales vascularizan la cabeza metatarsal, cuando existe compromiso en la zona subcondral, por estar aislada por una superficie convexa avascular del cartílago hialino se produce la necrosis avascular. Además, se sospecha que la posición anatómica de distintas entidades como son: la cabeza del metatarsiano, la cabeza femoral, el cóndilo humeral y el escafoides presentan vulnerabilidad y sensibilidad a la necrosis avascular y se relaciona con un cierre tardío de las epífisis de los metatarsianos que no concluye, hasta la edad de entre 17 a 20 años y esto genera compromiso en la circulación del hueso subcondral por la presencia de la placa de crecimiento ${ }^{11}$.

- Trauma: relacionado con la biomecánica y el daño preferente al segundo metatarsiano, a consecuencia de presentar un dedo más largo y rígido, por tanto, está sujeto a mayor compresión por el calzado en relación al eje longitudinal, produciendo el daño tisular, siendo la teoría más aprobada por los autores la de una sobrecarga que genera stress en la metáfisis distal del metatarsiano dando como resultado una fractura por stress ${ }^{12}$.

- Alteraciones sistémicas: son factores a tener en cuenta y que predisponen a la enfermedad los trastornos sistémicos como el lupus eritematoso sistémico, la hipercoagubilidad, que generan presión intraósea favoreciendo en la cabeza metatarsal el desarrollo del Freiberg ${ }^{13,14}$. 


\section{CLÍNICA}

Se presenta en la segunda década de la vida, en adolescentes que practican deporte, asociada comúnmente al género femenino y localizado principalmente en ${ }^{15,16}$ :

- $\quad$ Segundo metatarsiano $68 \%$ de personas.

- Tercer metatarsiano $27 \%$ de los casos y

- Bilateral en el 6,6\% de los casos.

La enfermedad de Freiberg es la única que afecta predominantemente a las mujeres con una relación mujer-hombre de 5:1 y suele ser unilateral ${ }^{17}$.

La sintomatología habitual que puede aparecer en las personas que padecen Freiberg se relaciona con cojera, dolor metatarsal que empeora al ponerse de pie y al caminar, claudicación espontánea, tumefacción dorsal, limitación del movimiento, aunque los síntomas pueden no comenzar hasta que la artritis degenerativa se ha desarrollado ${ }^{18,19}$.

En la exploración clínica, a la palpación se percibe dolor a la presión dorsoplantar de la cabeza metatarsiana afectada, con inflamación y limitación en la movilidad articular debido al dolor.

En casos crónicos, hay escasa movilidad, contracturas de los tejidos blandos periarticulares y aumento de volumen en la región dorsal del antepié, debido a la exostosis que se ha formado $^{20}$ (Figura 1).

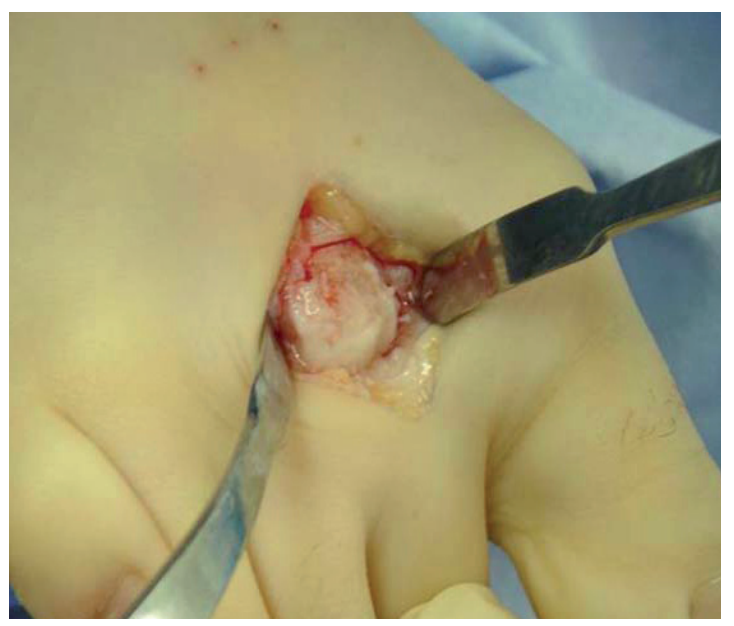

Figura 1. Aspecto intraoperatorio de la exostosis del segundo metatarsiano.

\section{DIAGNÓSTICO}

En estadíos iniciales el método diagnóstico más temprano es la gammagrafía ósea que hace visible la fragmentación subcondral y puede ser positiva hasta 6 meses antes de observarse alteraciones radiográficas ${ }^{21}$.

En el resto de etapas de presentación de la enfermedad, la clasificación se realiza atendiendo al examen radiológico e histopatológico que describió Smile en 5 etapas $^{22}$ :

- Fase I: se caracteriza por una fisura de la epífisis subcondral, sin interés radiológico debido a la ausencia de visibilidad del trastorno (Figura 2).

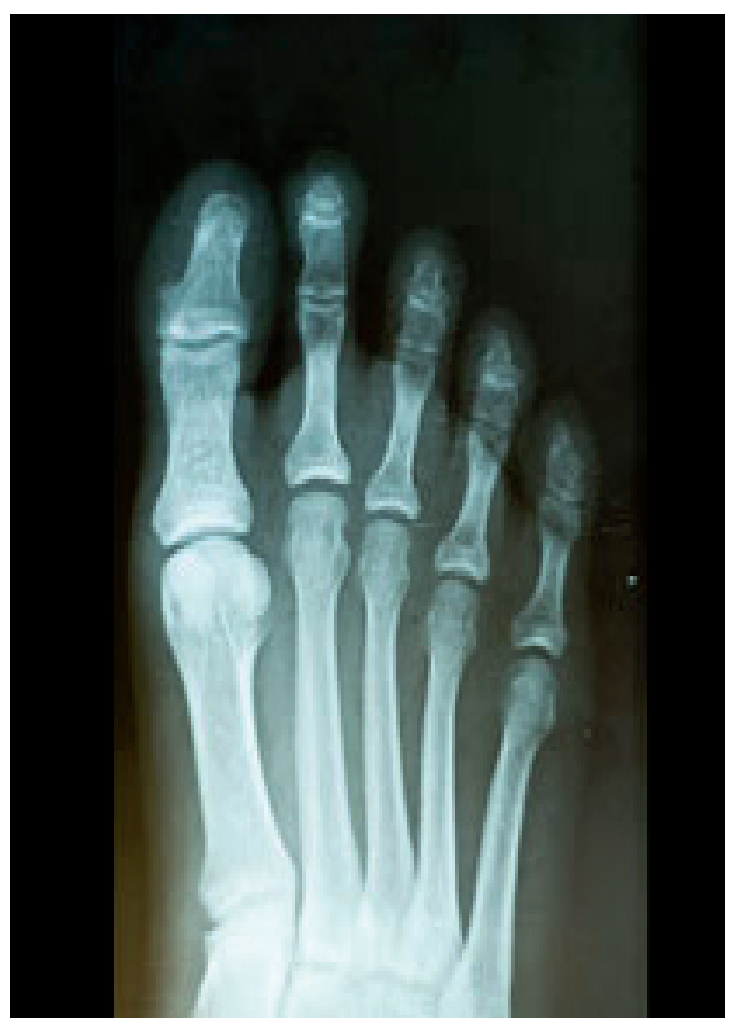

Figura 2. Aspecto radiológico de la enfermedad de Freiberg en el segundo metatarsiano en el estadío I.

En los estudios histopatológicos se observa esclerosis del hueso esponjoso adyacente a la línea de fractura y ausencia de aporte sanguíneo a la epífisis. 
- Fase II o fase de resorción ósea: da comienzo la revascularización produciéndose un colapso, en la zona central - dorsal de la cabeza metatarsal afectada que comienza a colapsarse hacia la metáfisis. En las radiografías oblícuas se evidencia de manera más clara el aumento del espacio articular, aplastamiento de la cabeza del metatarsiano, esclerosis leve de la epífisis y edema en los tejidos blandos (Figura 3).

- Fase III: se observan imágenes líticas alrededor de la fractura y un aumento de la esclerosis epifisiaria, que conllevan un aumento del aplanamiento y superficie en las regiones lateral y medial de la cabeza metatarsal con pérdida del espacio intraarticular (Figura 4).

- Fase IV: el hundimiento del fragmento central hacia la metáfisis conlleva a una pérdida de la superficie plantar, los bordes periféricos se separan originando cuerpos libres intraarticulares. La destrucción articular es irreparable y aparece una disminución del espacio articular en las radiografías (Figura 5).

- Fase V: se concluye la degeneración total de la articulación con eliminación del espacio articular, existe una hipertrofia de la cabeza metatarsal, acompañada de alteraciones de la base de la falange proximal, con la formación de osteófitos y engrosamiento de la diáfisis del metatarsiano a consecuencia del aumento en la carga diafisiaria al perderse la cabeza (Figura 6).

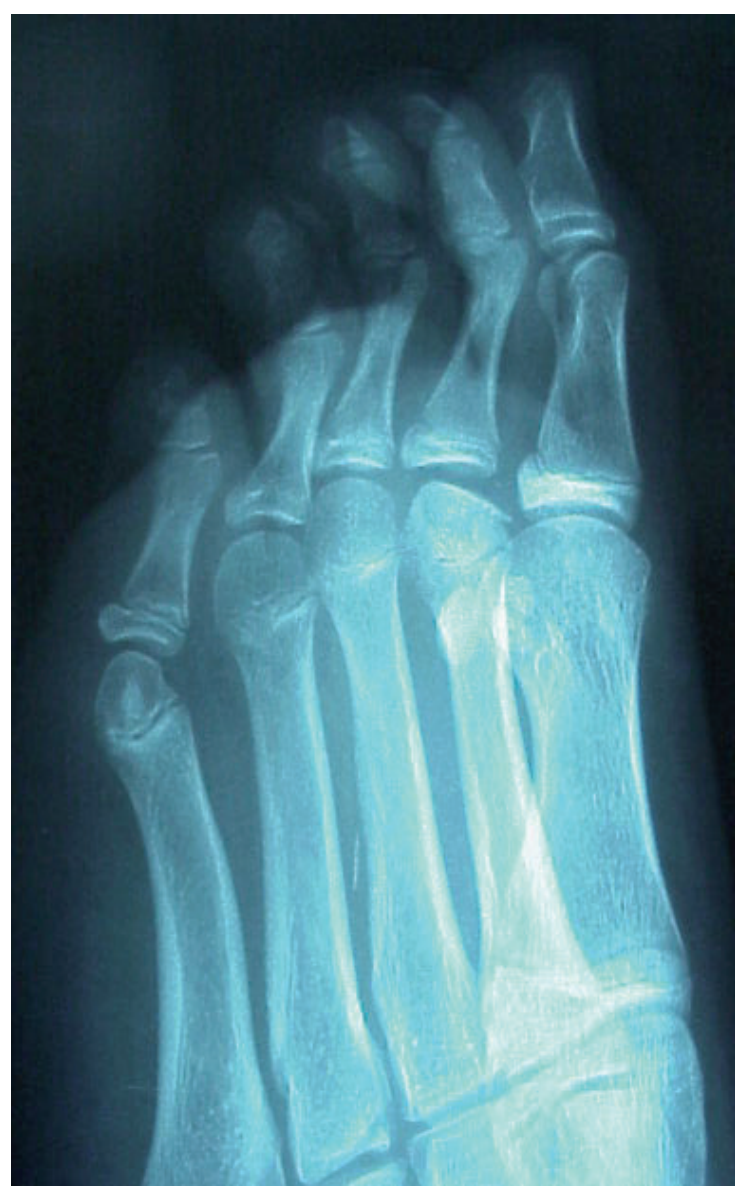

Figura 3. Radiografía oblícua del Freiberg en segundo metatarsiano en estadio II.

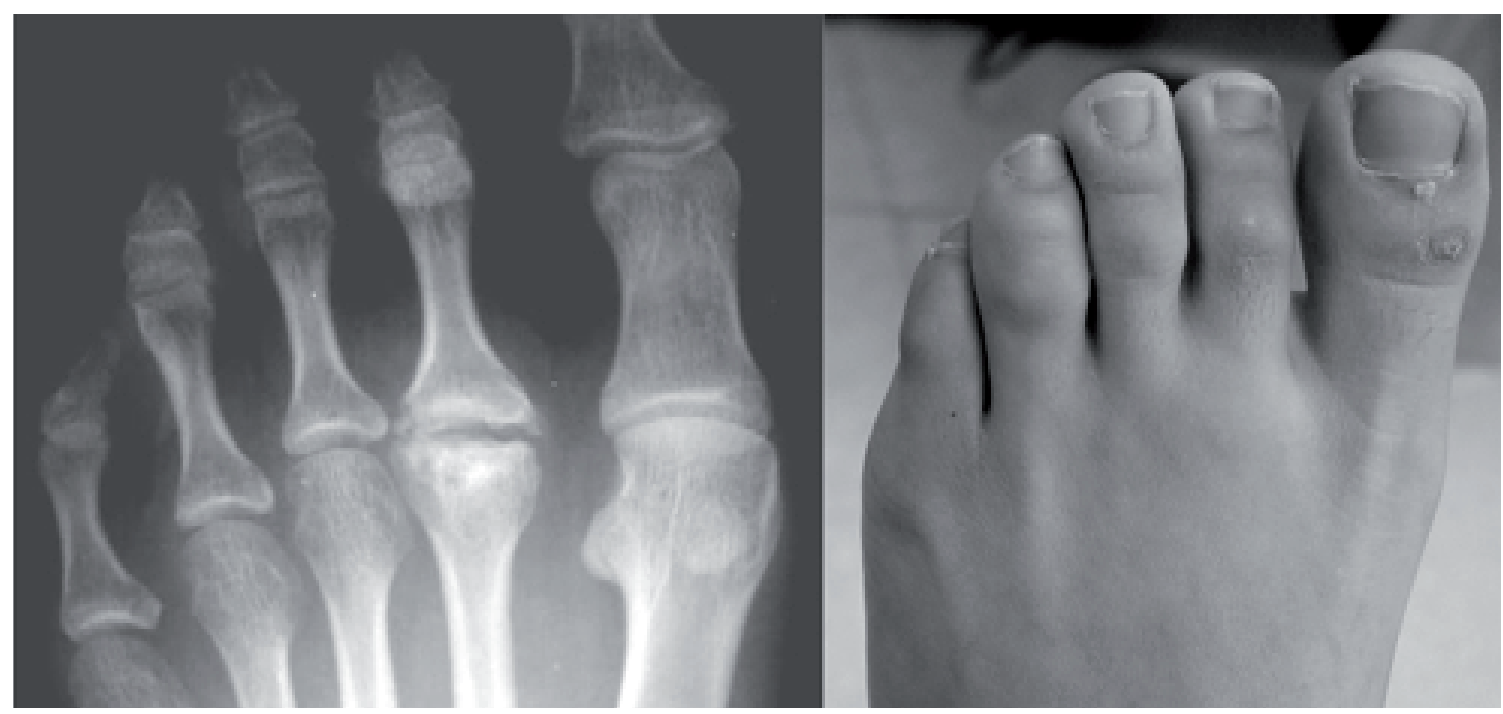

Figura 4. Radiografía con Freiberg en segundo metatarsiano en estadio III. 


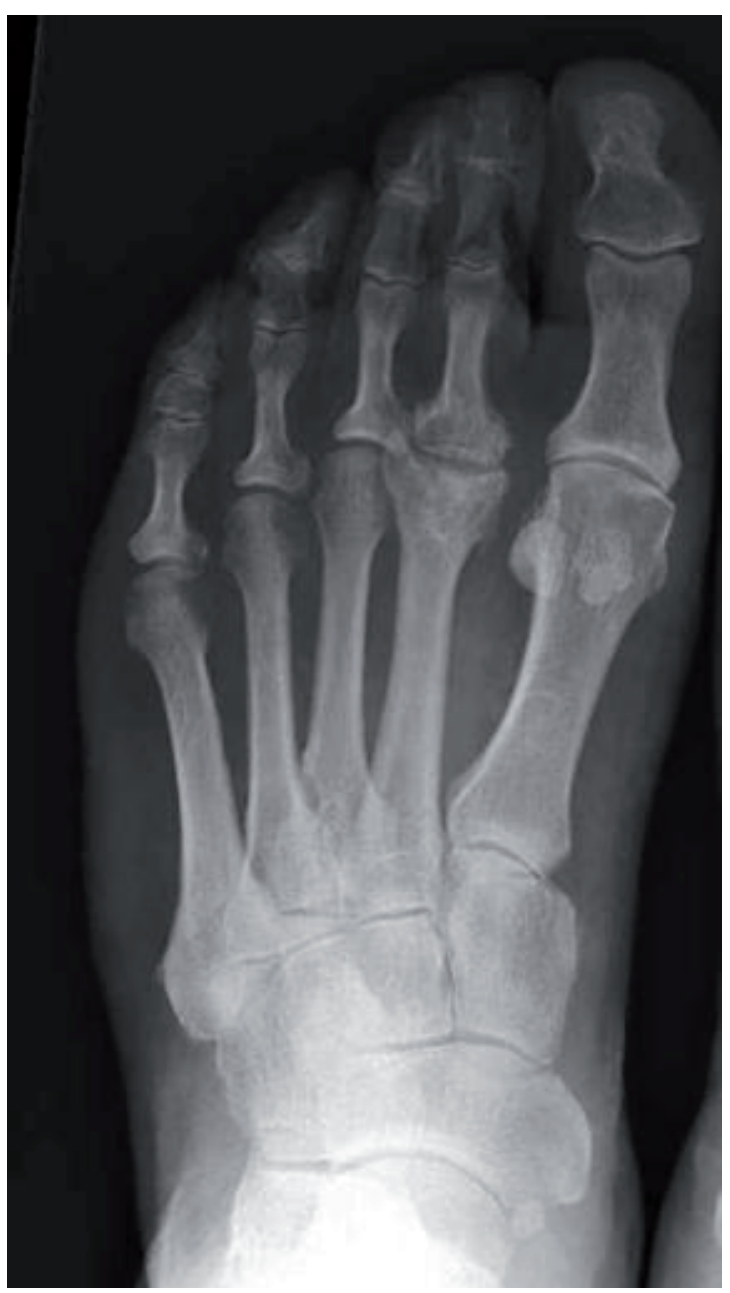

Figura 5. Radiografía en Freiberg en segundo metatarsiano en estadio IV.

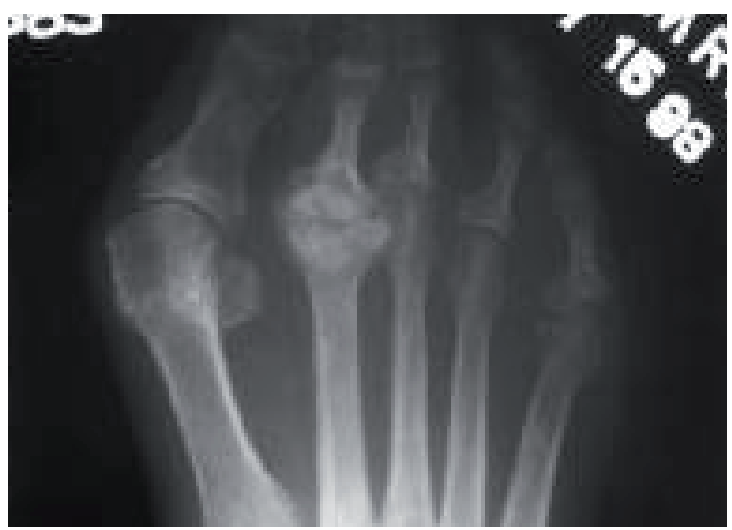

Figura 6. Radiografía oblicua del Freiberg en segundo metatarsiano en estadio IV.

\section{DIAGNÓSTICO DIFERENCIAL}

El diagnóstico diferencial puede ser difícil y se establece con fractura por estrés, artritis séptica, tumores, neuritis interdigital, sinovitis y diversos tipos de $\operatorname{artritis}^{23,24}$.

El diagnóstico es radiológico, encontrando osteoesclerosis en etapas tempranas y osteólisis en etapas más avanzadas, llegando a la destrucción progresiva de la cabeza del metatarsiano en la última fase de la enfermedad.

\section{TRATAMIENTO}

El tratamiento irá encaminado al igual que otras osteocondrosis a la gestión temprana de la enfermedad, para aliviar los síntomas y prevenir la deformidad ósea ${ }^{25}$.

En la mayoría de los casos, en los primeros estadíos de la enfermedad los pacientes responden positivamente a los tratamientos conservadores, sin expermientar secuelas a largo plazo $^{26}$.

Los tratamientos conservadores están relacionados con la fase clínica de la enfermedad e incluyen el uso de analgésicos, antiinflamatorios, reposo relativo, ortesis plantares, modificaciones en el calzado (suela gruesa, balancín, barra metatarsal), infiltración con esteroides (betametasona inyectable, dexametasona inyectable, triamcinolona inyectable...) y anestésicos que pueden disminuir parcialmente los síntomas y así evitar la progresión de la enfer$\operatorname{medad}^{27}$.

En aquellos casos, que los tratamientos conservadores son insuficientes y persiste el dolor, está indicado el manejo quirúrgico, para lo cual se han descrito diferentes procedimientos quirúrgicos que se puede dividir en dos categorías $^{19}$ :

1. Controlar la alteración de la fisiopatología y biomecánica anormal que pueden predisponer a un individuo a la enfermedad o empeorar el curso clínico. Estos incluyen la descompresión articular, la osteotomía metatarsiana correctiva, artroplastia de resección, osteotomía en dorsiflexión, en cuña se consigue paliar el incremento de la presión intra-ósea, que se asocia a la necrosis avascular y de este modo permitir la revascula- 
rización de la zona necrótica, antes de que se generen cambios estructurales y proceder a la curación de la enfermedad ${ }^{28,29}$.

2. Reparar la congruencia articular o tratar las características degenerativas encontradas en la etapas avanzadas de la enfermedad, por medio de un acortamiento del metatarsiano, artroplastia con prótesis de silicón, desbridamiento y perforación del cartílago por artroscopia, resección de cuerpos libres intraarticulare ${ }^{30,31}$.

En resumen y según Gautier el protocolo de actuación del Freiberg estará basado en el momento y el grado de evolución de la enferme$\operatorname{dad}^{27}$ (Tabla 1).

\section{CONCLUSIONES}

Aunque el descubrimiento de la enfermedad de Freiberg data del siglo pasado, la falta de consenso conlleva a que muchos clínicos no están familiarizados con el diagnóstico y el reto de proporcionar un tratamiento eficaz. Con base a nuestra revisión de la literatura, se recomienda que los siguientes aspectos clínicos, basados en la evidencia, sean una aproximación a los principios que deben guiar la evaluación y tratamiento de la enfermedad de Freiberg.

El diagnóstico de la enfermedad de Freiberg es clínico y se establece por la historia clínica, el examen físico, y las radiografías. La centellografía ósea es útil para establecer un diagnóstico precoz en el primer estadío de la enfermedad

Los tratamientos conservadores en las primeras etapas de la enfermedad consisten en el control de los síntomas y la prevención de la deformidad. Las opciones de tratamiento incluyen medicamentos antiinflamatorios, infiltraciones, inmovilización y/o reposo relativo, zapato de suela dura, ortesis plantares a medida y la mayoría de los pacientes responden positivamente a los tratamientos conservadores.

El tratamiento quirúrgico, está indicado si la sintomatología persiste después del mane-

Tabla 1. Clasificación y tratamiento de la enfermedad de Freiberg, según Gauthier.

\begin{tabular}{|c|c|c|}
\hline $\begin{array}{l}\text { Estadío de la } \\
\text { Enfermedad }\end{array}$ & Características & Tratamiento \\
\hline I & $\begin{array}{l}\text { Sin degeneración de articula- } \\
\text { ción. } \\
\text { Cartílago articular intacto. }\end{array}$ & $\begin{array}{l}\text { Ortesis a medida con almohadillo metatarsal. } \\
\text { Calzado con poco tacón. }\end{array}$ \\
\hline II & $\begin{array}{l}\text { Fragmentos periarticulares. } \\
\text { Cartílago articular intacto. }\end{array}$ & $\begin{array}{l}\text { Suprimir actividad deportiva. } \\
\text { Queleictomía. } \\
\text { Desbridamiento eliminación de cuerpos } \\
\text { libres, resección de osteofitos periféricos y } \\
\text { sinovectomía. }\end{array}$ \\
\hline III & $\begin{array}{l}\text { Degeneración severa articular. } \\
\text { Pérdida de cartílago articular. }\end{array}$ & $\begin{array}{l}\text { Du Vries. } \\
\text { Artoplastia resección parcial de la articula- } \\
\text { ción metatarsofalángica, incluyendo cóndilos } \\
\text { plantares y en casos de hipertrofia hasta la } \\
\text { base proximal de la falange. }\end{array}$ \\
\hline
\end{tabular}

IV Displasia Epifisaria

Displasia Epifisaria.
Fragmentación múltiple de la Las indicaciones de estadios I, II y II. cabeza. 
jo conservador. Se han descrito diversas técnicas quirúrgicas, principalmente variedades de osteotomías, pero no se ha comprobado la mejoría en la circulación de la cabeza metatarsal, probablemente la descompresión articular, incluso con técnica artroscópica, favorezca la mejoría en cuanto a la circulación distal del metatarsiano, pero tiene aplicación únicamente en estadios iniciales, en los que no se establece el diagnóstico en la mayoría de los casos, siendo la técnica de remodelación de la cabeza metatarsal, la que ha dado buenos resultados en los pacientes sometidos a intervención quirúrgica.

\section{REFERENCIAS}

1. Smith TWD, Kreibich DN. Freiberg's Disease. In Hallux Valgus and forefoot surgery, Hetherington, VJ (Ed), Churchill Livingstone, New York 1988. p.453-457.

2. Gómez M, Santamaría, E. Enfermedad de Freiberg. Rev Esp Podol 2006, 17 (5): 226-233.

3. Fernández JM, Justino L, Camacho J. Enfermedad de Freiberg: Osteocondrosis de la cabeza del segundo metatarsiano. An Med Asoc Med Hosp ABC 2002; 47 (2): 81-84.

4. Banks AS. Avascular necrosis of the first metatarsal head. A different perspective. J Am Podiatr Med Assoc 1999; 89: 441-453.

5. Kline L. Freiberg's Infraction of the Second Metatarsal Head with Bioorthologic Repair using the NEXA Osteocure ${ }^{\mathrm{TM}}$ Bone Graft: A case report. The Foot \& Ankle Journal 2008; 1 (11): 4.

6. Hyun-Sik G, Kyung-Hak, L, Joo Han O. Fixation of dorsla wedge osteotomy forFreiberg's disease using bioabsorbable pins. Foot \& Ankle International 2003; 24(11): 876-877.

7. Freiberg AH. Infraction of the second metatarsal bone, a typical injury. Surg Gyn Ob 1914; 19:191-163.

8. Chun KA, Oh HK, Wang KH, Suh JS. Freiberg's disease: quantitative assessment of osteonecrosis on three-dimensional CT. J Am Podiatr Med Assoc 2011, 101 (4), 335-40.

9. Del Prado M, Ripoll P, Golanó, P. Cirugía percutánea del pie. Barcelona: Masson, 2003.

10. Melé J, Villanueva M, Villalba L. Enfermedad de Freiberg bilateral: a propósito de un caso. Form Med Contin Aten Prim. 2007; 14 (5): 275-6.

11. Crock HV: The Blood Supply of the Lower Limb Bones in Man. Livingstone Ltd., Edinburgh and London, 1967.

12. Stanley D, Betts RP, Rowley DI, Smith TW. Assessment of etiologic factors in the development of Freiberg's disease. J Foot Surg 1990; 29 (5): 444-447.

13. Lai KA, Shen WJ, Yang CY, Shao CJ, Hsu JT, Lin RM. The use of alendronate to prevent early collapse of the femoral head in patients with non traumatic osteonecrosis. A Randomised clinical study. $\mathrm{J}$ Bone Joint Surg Am 2005, 87(10): 2155-9.

14. Lieberman JR, Berry DJ, Mont MA, Aaron RK, Callaghan JJ, Rajadhyaksha AD, Urbaniak JR. Osteonecrosis of the hip: management in the 21st century. Instr Course Lect 2003; 52: 337-55.

15. Enriquez C, Guevara H, Estevez D.Plastia de interposicion en el tratamiento de osteocondritis del segundo metatarsiano. Presentacion de un caso. Acta Ortop Mex. 2008 Jul-Aug; 22(4):259-62.

16. Contreras MA. Osteocondrosis de la cabeza del segundo metatarsiano o enfermedad de Freiberg. Rev Esp Enferm Metab Oseas. 2003; 12 (1): 12:40.

17. Katcherian D.A. Treatment of Freiberg's disease. Orthop Clin North Am 1994; 25 (1), 69-81.

18. Alan T, Shih AT, Quint, RE, Armstrong, DG, Nixon, BP. Treatment of Freiberg's Infraction with the Titanium Hemi-implant. J Am Podiatr Med Assoc 2004, 94 (6): 590-593.

19. Carmont MR. Current concepts review: Freiberg's disease. Foot Ankle Int 2009; 30:167.

20. King-Hayata, MA, Martínez-de Anda MC, King-Martínez M, King-Martínez AC, Cuéllar-Avaroma A. Tratamiento de la enfermedad de Freiberg por cirugía percutánea: Reporte de un caso. Med Sur 2007; 14 (2), 75-79.

21. Smillie IS. Freiberg's infarction (Kohler's second disease). J Bone Joint Surg Br 1957; 39:580.

22. Smillie, I.S. Treatment of Freiberg's infraction. Proc R Soc Med 1967; 60: 29.

23. Mizel MS, Yodllowsky ML. Disorders of the lesser metatarsophalangeal joints. J Am Acad Orthop Surg 1995; 3: 166-173.

24. Scartozzi G, Schram A, Janigian J. Freiberg's infraction of the second metatarsal head with formation of multiple loose bodies. J Foot Surg 1989; 28(3): 195-199. 
25. Siffert RS. Classifcation of the osteochondroses. Clin Orthop 1981; 158:10.

26. Gillespy T, Genant HK, Helms CA. Magnetic resonance imaging of osteonecrosis. Radiol Clin North Am 1986; 24:193-208.

27. Gauthier G, Elbaz R. Freiberg's infraction: a subchondral bone fatigue fracture: a new surgical treatment. Clin Orthop 1979, 42, 93.

28. Dolce M; Osher L; McEneaney P; Prins D. The use of surgical core decompression as treatment for avascular necrosis of the second and third metatarsal heads. The Foot 2006; 17:162-166.

29. Frieberg AA; Freiberg RA; Core decompression as a novel treatment for early Freiberg's infraction of the second metatarsal head. Orthopedics 1995; 18(12):1177-1178.

30. Salvi AE, Metelli GP: A case of Freiberg's disease in an adult patient. Chir Organi Mov 2004; 325-328.

31. Sproul J, Klaaren H, Mannarino F. Surgical treatment of Freiberg's Infraction in athletes. Am J Sports Med 1993; 21:381-384. 